\title{
Development and validation of the 10-item Social Provisions Scale (SPS-10) Japanese version
}

Fujika Katsuki ( $\nabla$ katsuki@med.nagoya-cu.ac.jp )

Nagoya City University https://orcid.org/0000-0002-0822-4776

Atsurou Yamada

Nagoya City University

Masaki Kondo

Nagoya City University

Hanayo Sawada

Nagoya City University

Norio Watanabe

Kyoto University

Tatsuo Akechi

Nagoya City University

Paola Rucci

University of Bologna

\section{Research note}

Keywords: Social support, Social provisions scale, Validity, Reliability

Posted Date: April 2nd, 2020

DOI: https://doi.org/10.21203/rs.3.rs-19247/v1

License: (c) (i) This work is licensed under a Creative Commons Attribution 4.0 International License.

Read Full License 
Title: Development and validation of the 10-item Social Provisions Scale (SPS10) Japanese version

\section{Authors}

Fujika Katsuki, RN, PhD (Corresponding author)

Department of Psychiatric and Mental Health Nursing, Nagoya City University Graduate School of Nursing, Nagoya, Japan

467-8601, 1 Kawasumi, Mizuho-cho, Mizuho-ku, Nagoya, Japan

katsuki@med.nagoya-cu.ac.jp

Phone: $\quad+81-52-853-8048$

Fax: $\quad+81-52-853-8048$

Atsurou Yamada, MD, PhD

Department of Psychiatry and Cognitive-Behavioral Medicine, Nagoya City University Graduate School of Medical Sciences, Nagoya, Japan

1 Kawasumi, Mizuho-cho, Mizuho-ku, Nagoya, Japan

atsurou@med.nagoya-cu.ac.jp

Masaki Kondo, MD, PhD

Department of Psychiatry and Cognitive-Behavioral Medicine, Nagoya City University Graduate School of Medical Sciences, Nagoya, Japan

1 Kawasumi, Mizuho-cho, Mizuho-ku, Nagoya, Japan

kondo-masaki@umin.ac.jp

Hanayo Sawada, RN, MSN

Department of Psychiatric and Mental Health Nursing, Nagoya City University Graduate School of Nursing, Nagoya, Japan 
467-8601, 1 Kawasumi, Mizuho-cho, Mizuho-ku, Nagoya, Japan

hanayo874874@gmail.com

Norio Watanabe, MD, PhD

Department of Health Promotion and Human Behavior, Kyoto University Graduate School of Medicine/School of Public Health, Kyoto, Japan

Yoshida Konoe-cho, Sakyo-ku, Kyoto, Japan

noriowncu@gmail.com

Tatsuo Akechi, MD, $\mathrm{PhD}$

Department of Psychiatry and Cognitive-Behavioral Medicine, Nagoya City University Graduate School of Medical Sciences, Nagoya, Japan

1 Kawasumi, Mizuho-cho, Mizuho-ku, Nagoya, Japan

takechi@med.nagoya-cu.ac.jp

Paola Rucci, $\mathrm{PhD}$

Department of Biomedical and Neuromotor Sciences, Alma Master Studiorum, University of Bologna, Bologna, Italy

Via San Giacomo12, Bologna, Italy

paola.rucci2@unibo.it

Figures 2, Tables 3 


\section{Abstract (200 words)}

Objective: The Social Provisions Scale is one of the most widely used measures of perceived social support. Our aim was to develop and validate the Japanese version of the 10-item Social Provisions Scale (SPS-10) to be able to evaluate the social support of individuals easily in Japan. Results: The English version of the SPS-10 questionnaire was translated to Japanese and crossculturally adapted. The SPS-10 Japanese version was administered to 72 mothers (age, mean \pm standard deviation, $55.1 \pm 6.7$ years) of patients with eating disorders. The construct validity of the SPS-10 Japanese version was examined by comparing their scores with the scores on other scales: Pearson's correlation coefficient was -0.79 with the UCLA Loneliness Scale, -0.44 with the Beck Depression Inventory, and -0.39 with the K6. Cronbach's $\alpha$ as a measure of internal consistency was good at 0.89 . Structural validity was tested using confirmatory factor analysis. The confirmatory factor analysis model showed satisfactory goodness-of-fit indices except for RMSEA (CFI $=0.894$, GFI $=0.871$, AGFI $=0.716$, RMSEA=0.14). All items had high loadings on the respective factors, ranging from 0.48 to 0.88 . Our findings supported the construct validity, structural validity, and reliability of the SPS-10 Japanese version in Japanese people as a measure of social provisions.

Key words: Social support, Social provisions scale, Validity, Reliability 


\section{(1980 words)}

\section{Introduction}

Having positive supportive relationships with family and friends, who can listen and help in the event of problems, has a beneficial effect on maintaining psychological health. A large number of studies have investigated the link between social support and depressive symptoms [1]. Clara et al. reported that having higher perceived available social support was associated with fewer depressive symptoms [2]. Several prospective studies have found that positive support from family members can facilitate recovery from a major depressive episode $[3,4]$. Some studies have reported that greater social support deficits predict subsequent higher depressive symptomatology $[5,6]$. Therefore, in research studies associated with mental health and social support, it is important to develop a scale that can appropriately measure social support and be compared internationally.

The Social Provisions Scale (SPS) developed by Cutrona and Russell [7] is one of the most widely used measures of perceived social support. The SPS is a 24-item self-report questionnaire, and has the following six subscales that measure six types of social provision: attachment, reassurance of worth, reliable alliance, social integration, guidance, and opportunity for nurturance. The measure has good psychometric properties in term of reliability and predictive, convergent and construct validity $[7,8]$. The measure has been used in diverse populations such as college students [9], spouses of cancer patients [10], and patients with multiple sclerosis [11]. The 10-item Social Provisions Scale (SPS-10) is a short version of the original SPS and was developed by Iapichino et al.[12] in Italian. The reliability and validity of the Italian SPS-10 are excellent.

The aim of the present study was to develop the SPS-10 Japanese version and to examine its reliability and validity. 


\section{Main text}

\section{Subjects and Procedure}

The study population consisted of mothers of patients with an eating disorder. The mothers of patients with eating disorder were selected as subjects because the family burden of patients with eating disorders is high. Potential subjects were recruited from four family self-help groups of patients with eating disorders in the community and one university hospital in Japan from July 2017 to August 2018. Inclusion criteria were: the mother of a female patient who met the criteria for the clinical diagnosis of an eating disorder as determined by a physician in a hospital; the patient with an eating disorder was aged between 16 and 50 years; and the patient's mother was aged between 30 and 85 years.

We gave participants of four family self-help groups of patients with eating disorders in the community and one university hospital a leaflet with an explanation of the purpose and procedure of the study. After receiving permission from mothers and the home address, we mailed questionnaires to their homes and enrolled them in the study when their completed questionnaires were returned.

\section{Measurements}

\section{Development of the 10-item Social Provisions Scale (SPS-10) Japanese version}

\section{SPS-10}

The Social Provisions Scale (SPS) assesses the degree to which an individual perceives that his/her social network provides different kind of social support, including opportunities for the individual to provide support.[7] The original SPS is a self-report questionnaire that includes 24 items rated on a four-point Likert-type scale. The scale includes the following six subscales based on Weiss's social provisions theory [13]: reliable alliance, attachment, opportunity for 
giving support to others, social integration, reassurance of worth, and guidance.

Iapichino et al. [12] developed a short version of the SPS, which consists of 10 items (SPS-10). The SPS-10 retains the following five of the six original SPS subscales: attachment (emotional support), social integration, reassurance of worth, reliable alliance (material support) and guidance. In the original SPS, each subscale has four items: two positively worded items describing the presence of a type of support and two negatively worded items assessing the absence of a type of support. The SPS-10 has only negatively worded items, and the total score on the SPS-10 ranges from 10 to 40 [12]. A higher total score indicates stronger perceived provision of social support.

\section{Translation}

Two native Japanese speakers translated into Japanese independently the 10 items of the English version of the original SPS that corresponded to the items in the Italian version of the SPS-10. One of the translators was a psychiatrist (NW), while the other was a psychiatric nurse (FK). The two translators met to discuss the forward-translated Japanese version. Two other translators who were blind to the original version translated the Japanese-translated version back into English in order to ascertain whether the translated questions communicate comparable meanings. One of the translators spoke both Japanese and English, while the other was a Japanese science translator specializing in psychiatry and psychology. Finally, the original author of the SPS-10 Italian version (PR) confirmed semantic and conceptual equivalence among the SPS-10 Italian version and the original English version and the backtranslated English version. The SPS-10 Japanese version is a 10-item self-report questionnaire with higher scores indicating higher social support experience. Each item is rated on a fourpoint Likert-type scale ranging from 1 to 4 . 


\section{UCLA Loneliness Scale (ULS)}

The ULS is a self-report questionnaire developed by Russell [14]. The ULS contains 20 items, with higher scores indicating higher loneliness experience. The reliability and validity of the Japanese version of the ULS are excellent [15]. Cronbach's $\alpha$ coefficient of reliability in this study was 0.90 .

\section{Beck Depression Inventory (BDI-II)}

The BDI-II is a 21-item self-report instrument that assesses the presence and severity of symptoms of depression [16]. The reliability and validity of the Japanese version of the BDI-II are excellent [17]. Cronbach's $\alpha$ coefficient of reliability in this study was 0.92 .

\section{K6}

We used the K6 questionnaire, a six-item self-report questionnaire that was developed to screen for $D S M-I V$ defined depression and anxiety disorders within 30 days prior to its administration. A validation study of the Japanese version of the K6 questionnaire showed that the K6 has excellent validity [18]. Cronbach's $\alpha$ coefficient of reliability in this study was 0.86 .

\section{Statistical analysis}

Descriptive data analysis was conducted by calculating frequencies, median, mean scores, and standard deviation. To determine the construct validity of the SPS-10 Japanese version, we calculated Pearson's correlation coefficients between the total score on the SPS-10 Japanese version and the scores of the other scales. The internal consistency of the SPS-10 Japanese version was tested using Cronbach's $\alpha$ coefficient. In addition, to investigate the structural validity of the scale, confirmatory factor analysis of items was carried out. Each item was 
specified to load onto only the factor it was designed to measure, with correlations among the five factors freely estimated. In order to test the goodness of fit of the model, four approximate fit indices were considered: comparative fit index (CFI); goodness fit index (GFI); adjusted GFI (AGFI), with values $>0.8$ and 0.9 indicating acceptable and excellent fit, respectively; and root mean square error of approximation (RMSEA) with values $<0.05$ and 0.08 indicating close and reasonable fit, respectively. Statistical analysis was performed using SPSS Statistics version 22 and SPSS Amos version 26.

\section{Ethical Issues}

This study was approved by the Ethics Review Committee of Nagoya City University Graduate School of Medical Sciences, Japan (Ref: No 60-17-0001), and was conducted in accordance with the principles stated in the Helsinki Declaration. The study is registered at University Hospital Medical Information Network under number UMIN000031698 (March 9, 2018). Participants were given an explanation of the purpose and the procedure of the study using a leaflet. After receiving permission from mothers and the home address, we mailed questionnaires to their homes and enrolled them in the study when their completed questionnaires and their written consent were returned.

\section{Results}

The study sample consisted of 72 mothers of patients with an eating disorder who completed the SPS-10 Japanese version questionnaire, ULS, BDI-II and K6. The disease duration of the patients was 7.6 [standard deviation $(\mathrm{SD})=5.5$ ] years. Table 1 summarizes the demographic characteristics and clinical status of the subjects. Table 2 shows the frequency distribution of the SPS-10 Japanese version scores, that was slightly asymmetric. 
Cronbach's $\alpha$ for the overall scale was 0.89 , denoting a good internal consistency. The total score of the SPS-10 Japanese version had significant negative correlations with three other scales: $r=-0.79$ (Pearson correlation coefficient) $(\mathrm{p}<0.01)$ with the ULS, $r=-0.44(p<0.01)$ with the BDI-II, and $\mathrm{r}=-0.39(\mathrm{p}<0.01)$ with the K6.

We conducted a confirmatory factor analysis assuming a five-factor model with the five subscales of attachment, social integration, reassurance of worth, reliable alliance and guidance (Figure 1). The confirmatory factor analysis model showed satisfactory goodness of fit indices except for the RMSEA (CFI $=0.894$, GFI $=0.871$, AGFI $=0.716$, and RMSEA=0.14). All items had high loadings on the respective factors, ranging from 0.48 to 0.88 .

\section{Discussion}

The present study is the first to develop the SPS-10 Japanese version and to examine its reliability and validity. The results indicated that the SPS-10 Japanese version possesses excellent psychometric properties (Pearson's correlation coefficient between the SPS-10 Japanese version and the UCLA loneliness scale was -0.79). The participants of this study were mothers of patients with eating disorders whose mean duration of treatment for their disease was $7.6(\mathrm{SD}=5.5)$ years. The family burden of patients with eating disorders is high because family members are over-involved in behavioral and emotional aspects[19]. The participants of this study had somewhat worse mental health status than the general population in Japan [K6 scores in our participants $6.9 \pm 4.2$ versus $3.6 \pm 3.9[20]$; BDI-II score in our participants 14.1 \pm 9.7 , indicating mild depressive symptoms], and more than half of them had received counseling themselves (Table 1). Therefore, the participants of this study needed some support and might be an appropriate group to assess the validity and reliability of the SPS-10 Japanese version which evaluates social support. 
The frequency distribution of the scores on the SPS-10 Japanese version was similar to that of the SPS-10 Italian version (Table 2). The value of Cronbach's $\alpha$ obtained in the present study was 0.89 and was similar to the internal consistency coefficient obtained in the Italian study on the SPS-10 (0.809) [12]. This lends support for excellent internal consistency and reliability of the SPS-10 Japanese version and suggests that this scale can be used in Japanese people.

In the present study, the total score on the SPS-10 Japanese version was strongly negatively correlated with the ULS scores, which measures the degree of loneliness, and was moderately negatively corelated with the BDI-II score, which measures the degree of depression/anxiety. Weiss[13] reported that people need six types of social provisions, and if some type is deficient, people are at risk for experiencing social and/or emotional loneliness. This loneliness could subsequently lead to distress, tension, and disengagement, along with depression. Our findings suggest good construct validity of the SPS-10 Japanese version.

We performed confirmatory factor analysis using the five factors of the SPS-10 Italian version[12] and confirmatory factor analysis model had a good fit to data. All items had sufficient factor loading on their respective factors $(0.48-0.88)$. These results suggest that the structure of the SPS-10 Japanese version was consistent. Our findings suggest that the SPS-10 Japanese version has good reliability and validity as a measure of social support in the Japanese population.

In conclusion, the results of this study supported the construct, structural validity and reliability of the SPS-10 Japanese version in a sample of Japanese women. The SPS-10 Japanese version is simple and easy to use. We demonstrated the potential utility of the SPS-10 Japanese version as a screening tool to assess social support in people experiencing difficulties in Japan. 


\title{
Limitations
}

The current study has some limitations. First, the participants in this study were only women and were family members of patients with an eating disorder. Because the purpose of this study was to develop and validate a scale for measuring social support, it is appropriate that the participants had some difficulty, but families of patients with an eating disorder were a specific sample. Second, the sample size was small. Further research using large samples is needed.

\begin{abstract}
Abbreviations
SPS: Social Provisions Scale; ULS: UCLA Loneliness Scale; BDI: Beck Depression Inventory; CFI: comparative fit index; GFI: goodness fit index; AGFI: adjusted GFI; RMSEA: root mean square error of approximation
\end{abstract}

\section{Acknowledgments}

The authors wish to thank Takao Suzuki of the family support group of eating disorder 'Pokoapoko' and Riho Sato for support in this study. We thank all participants of this study.

\section{Author Contributions}

FK, AY, HS and NW participated in designing the study. FK, NW and PR contributed to translation process. FK wrote the paper. FK and AY conducted recruitment and data collection. NW, MK, AT and PR supervised the study and edited various drafts of the paper. All authors have read and approved the final manuscript. 


\section{Funding}

This study was supported by a Grant-in-Aid for Scientific Research KAKENHI Grant Numbers 16K12256 from the Japanese Ministry of Education, Science, and Technology.

\section{Availability of data}

The datasets used and/or analyzed during the current study are available from the corresponding author on reasonable request.

\section{Ethics approval and consent to participate}

This study was approved by the Ethics Review Committee of Nagoya City University Graduate School of Medical Sciences, Japan (Ref: No 60-17-0001). All participants provided written informed consent to the study.

\section{Consent to publish}

Not applicable.

\section{Competing interests}

HS and PR declare no conflicts of interest. FK received speaker's fees from Otsuka Pharmaceutical CO., LTD. AY received medical fees from Gifu Hospital, speaker's fees from Aichi Education and Sports foundation, the family support group of autism spectrum disorders 'Hogaraka', Kyowa Pharmaceutical Industry, Meiji Seika Pharma Co., Ltd, Mental Care Association Japan, Mochida Pharmaceutical CO., LTD., Otsuka Pharmaceutical CO., LTD., Shionogi \& Co., Ltd., other fees from Nagoya City. MK reports grant from the Japanese Ministry of Education, Science, and Technology, grants from Novartis Pharma K.K., personal 
fees from SHIONOGI \&CO., LTD., personal fees from Yoshitomiyakuhin Corporation, outside the submitted work. TA has received lectures fees from Astellas, AstraZeneca, DaiichiSankyo,Dainippon-Sumitomo, Eisai, Hisamitsu, Janssen, Kyowa-hakko Kirin, Kyowa, Lilly, MSD, Meiji-seika Pharma, Mochida, Mundipharma, Nipro, Otsuka, Pfizer, Shionogi, Terumo, and Tsumura.TA has received research funds from Daiichi-Sankyo, Eisai, FUJIFILM RI Pharma, Lilly, MSD, Novartis, Otsuka, Shionogi, and Tanabe-Mitsubishi. NW has received royalties from Sogensha, Medical view and Advantage Risk Management for writings.

$+$

\section{References}

1. Ibarra-Rovillard MS, Kuiper NA: Social support and social negativity findings in depression: perceived responsiveness to basic psychological needs. Clinical psychology review 2011, 31(3):342-352.

2. Clara IP, Cox BJ, Enns MW, Murray LT, Torgrudc LJ: Confirmatory factor analysis of the multidimensional scale of perceived social support in clinically distressed and student samples. Journal of personality assessment 2003, 81(3):265-270.

3. George LK, Blazer DG, Hughes DC, Fowler N: Social support and the outcome of major depression. Br J Psychiatry 1989, 154:478-485.

4. Keitner GI, Ryan CE, Miller IW, Norman WH: Recovery and major depression: factors associated with twelve-month outcome. Am J PSychiatry 1992, 149(1):93-99.

5. Leskela U, Rytsala H, Komulainen E, Melartin T, Sokero P, Lestela-Mielonen P, Isometsa E: The influence of adversity and perceived social support on the outcome of major depressive disorder in subjects with different levels of depressive symptoms. Psychol Med 2006, 36(6):779-788.

6. Nasser EH, Overholser JC: Recovery from major depression: the role of support from family, 
friends, and spiritual beliefs. Acta Psychiatr Scand 2005, 111(2):125-132.

7. Cutrona CE, Russell D: The provision of social relationship and adaptation to stress. Advances in Personal relationships 1987, 1:37-67.

8. Perera HN: Construct Validity of the Social Provisions Scale: A Bifactor Exploratory Structural Equation Modeling Approach. Assessment 2016, 23(6):720-733.

9. Perera HN, McIlveen P, Burton LJ, Corser DM: Beyond congruence measures for the evaluation of personality factor structure replicability: An exploratory structural equation modeling approach. Personality and Individual Differences 2015, 84:23-29.

10. Baron RS, Cutrona CE, Hicklin D, Russell DW, Lubaroff DM: Social support and immune function among spouses of cancer patients. J Pers Soc Psychol 1990, 59(2):344-352.

11. Chiu CY, Motl RW, Ditchman N: Validation of the Social Provisions Scale in people with multiple sclerosis. Rehabilitation psychology 2016, 61(3):297-307.

12. Iapichino E, Rucci P, Corbani IE, Apter G, Quartieri Bollani M, Cauli G, Gala C, Bassi M: Development and validation of an abridged version of the Social Provisions Scale (SPS-10) in Italian. Journal of Psychopathology 2016, 22:157-163.

13. Weiss R: The provisions of social relationships. In: Doing unto others. edn. Edited by Rubin Z. Engelwood Cliffs, NJ: Prentice Hall; 1974: 17-26.

14. Russell DW: UCLA Loneliness Scale (Version 3): reliability, validity, and factor structure. Journal of personality assessment 1996, 66(1):20-40.

15. Moroi K: Dimensions of the revised UCLA Loneliness Scale Annual reports of Departments of Social \& Human Studies and Language \& Literature 1992, 42:23-51.

16. Beck AT, Steer RA, Brown GK: Manual for the Beck Depression Inventory Second edition(BDI-II). San Antonia, TX: The Psychological corporation; 1996.

17. Hiroe T, Kojima M, Yamamoto I, Nojima S, Kinoshita Y, Hashimoto N, Watanabe N, Maeda 
T, Furukawa TA: Gradations of clinical severity and sensitivity to change assessed with the Beck Depression Inventory-II in Japanese patients with depression. Psychiatry Res 2005, 135(3):229-235.

18. Furukawa TA, Kawakami N, Saitoh M, Ono Y, Nakane Y, Nakamura Y, Tachimori H, Iwata $\mathrm{N}$, Uda H, Nakane $\mathrm{H}$ et al: The performance of the Japanese version of the $\mathrm{K} 6$ and $\mathrm{K} 10$ in the World Mental Health Survey Japan. Int J Methods Psychiatr Res 2008, 17(3):152-158.

19. Dimitropoulos G, Carter J, Schachter R, Woodside DB: Predictors of family functioning in carers of individuals with anorexia nervosa. Int J Eat Disord 2008, 41(8):739-747.

20. Sakurai K, Nishi A, Kondo K, Yanagida K, Kawakami N: Screening performance of K6/K10 and other screening instruments for mood and anxiety disorders in Japan. Psychiatry Clin Neurosci 2011, 65(5):434-441.

\section{Figure Legends}

Figure 1. Results from confirmatory factor analysis 
Figures

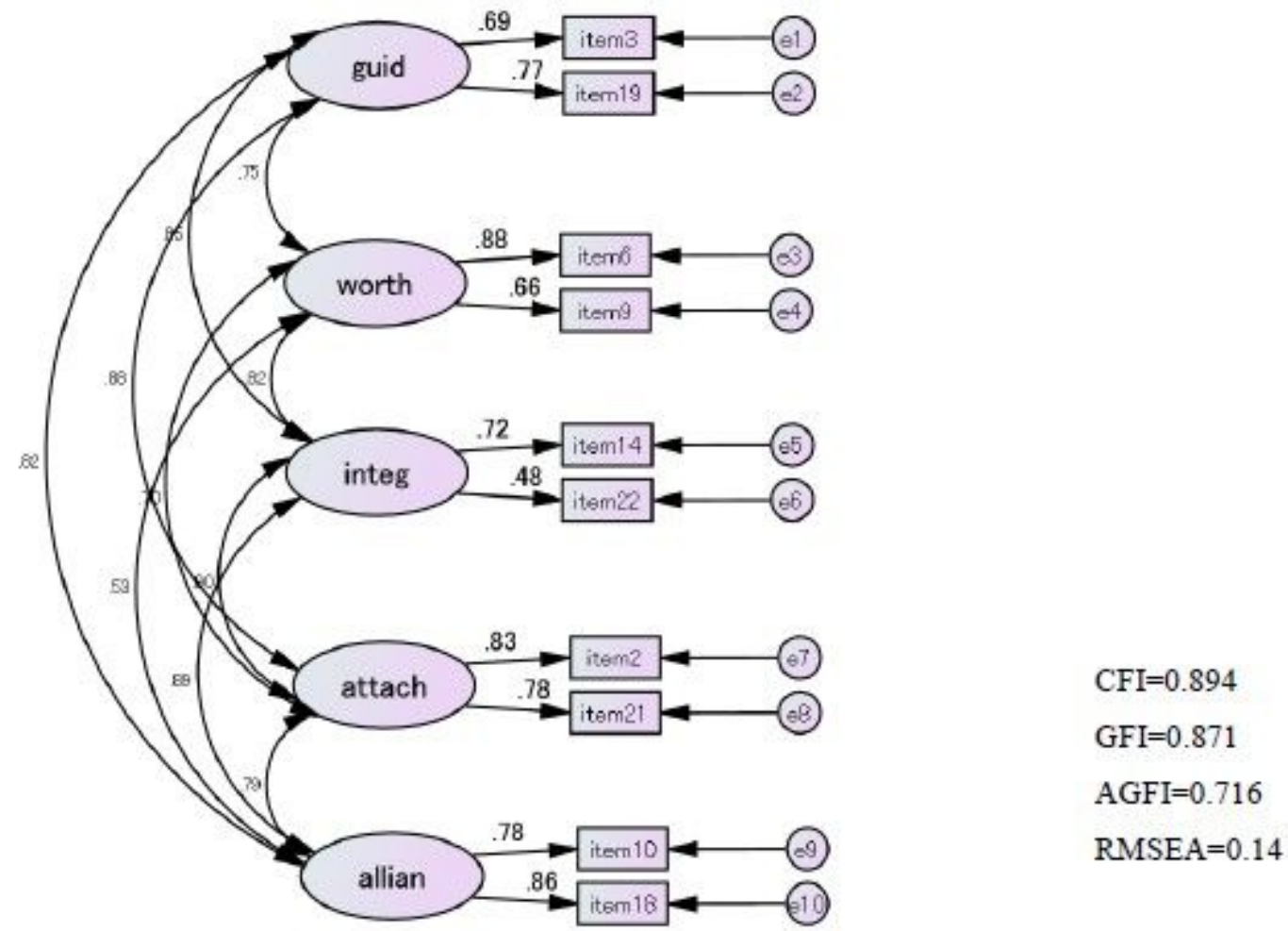

Abbreviations: guid; guidance, worth; reassurance of worth, integ; social integration, attach; attachment, allian; reliable alliance, CFI; comparative fit index, GFI; goodness fit index, AGFI; adjusted GFI, RMSEA; root mean square error of approximation

Figure 1

Results from confirmatory factor analysis

\section{Supplementary Files}


This is a list of supplementary files associated with this preprint. Click to download.

- Table12BMC.pdf 